\title{
Intraductal papillary mucinous neoplasm in an annular pancreas: a case report
}

\author{
Shinichiro Kobayashi ${ }^{1}$, Yukio Kamohara ${ }^{1 *}$, Yasuhiro Nagata', Masahiro Ito ${ }^{2}$ and Hikaru Fujioka ${ }^{1}$
}

\begin{abstract}
Annular pancreas is a rare anomaly in which a ring of pancreatic tissue encircles the second portion of the duodenum. We herein report a case involving a 79-year-old Japanese man with an intraductal papillary mucinous neoplasm (IPMN) of the pancreas. Imaging studies showed that the pancreatic tissue encircled the descending part of the duodenum and that a 30-mm-diameter cystic tumor was present in the annular segment, leading to the diagnosis of pancreatic IPMN. Limited pancreatic resection was successfully performed by careful division of the annular segment from the second portion of the duodenum. The postoperative course was uneventful, and the patient's pancreatic function was retained without the need for supplementation. To the best of our knowledge, this is the first report of IPMN occurring in the annular segment of the pancreas. Limited resection of the pancreatic annular segment is a feasible surgical treatment for noninvasive IPMN of the annular pancreas.
\end{abstract}

Keywords: Intraductal papillary mucinous neoplasm; Annular pancreas; Partial pancreatic resection

\section{Background}

Annular pancreas is a rare congenital anomaly caused by malrotation of the pancreatic ventral bud during embryonic development. This condition was first reported by Tiedemann in 1818 [1]. Autopsy and intraoperative studies have estimated the incidence of annular pancreas to be approximately 5 to 15 cases per 100,000 patients [2]. The coexistence of an annular pancreas with a pancreatic neoplasm is therefore exceptionally rare.

We herein report a case of an intraductal papillary mucinous neoplasm (IPMN) in an annular pancreas.

\section{Case presentation}

A 79-year-old Japanese man with epigastric pain was admitted to our hospital in January 2010. Abdominal ultrasonography revealed a large multilocular cystic mass (Fig. 1a). No intramural nodules or calcifications were present in the cystic lesion. Abdominal computed tomography showed that the pancreatic tissue encircled the descending part of the duodenum (Fig. 1b). A 30-mmdiameter cystic tumor was identified in the annular segment of the pancreas, and the tumor was linked to the

\footnotetext{
* Correspondence: ykamohara@nagasaki-mc.com

${ }^{1}$ Department of Surgery, National Hospital Organization Nagasaki Medical Center, Ohmura, Nagasaki, Japan

Full list of author information is available at the end of the article
}

annular duct. Magnetic resonance cholangiopancreatography also showed the annular duct encircling the duodenum (Fig. 1c), and the mass was linked to the main pancreatic duct. No intramural nodules or calcifications were detected in the tumor. The initial diagnosis was IPMN in the annular portion of the pancreas. These findings suggested a 30-mm-diameter branch-duct-type IPMN. Because the possibility of malignancy was not completely ruled out, we decided to remove the mass.

During surgery, we found that the pancreatic tissue encircled the second portion of the duodenum. The cystic mass was identified within the annular portion of the pancreas (Fig. 2a). Although loose adhesion of the annular portion of the pancreas to the duodenum was detected, the annular pancreas was carefully separated from the duodenum, preserving the serous membrane of the duodenum. All fibrotic bands between the duodenum and annular pancreas were ligated because small ducts between the duodenum and annular pancreas might be communicated with the pancreatic duct of the annular pancreas. Dissection of the annular portion (including the tumor) from the duodenum was completed without any injury to the duodenum. Frozen section examination of the surgical stump showed no malignancy. The distal side of the annular pancreas was dissected from the main pancreas using a linear endostapler (ENDO GIA

\section{实 Springer}

(c) 2015 Kobayashi et al. Open Access This article is distributed under the terms of the Creative Commons Attribution 4.0 International License (http://creativecommons.org/licenses/by/4.0/), which permits unrestricted use, distribution, and reproduction in any medium, provided you give appropriate credit to the original author(s) and the source, provide a link to the Creative Commons license, and indicate if changes were made. 

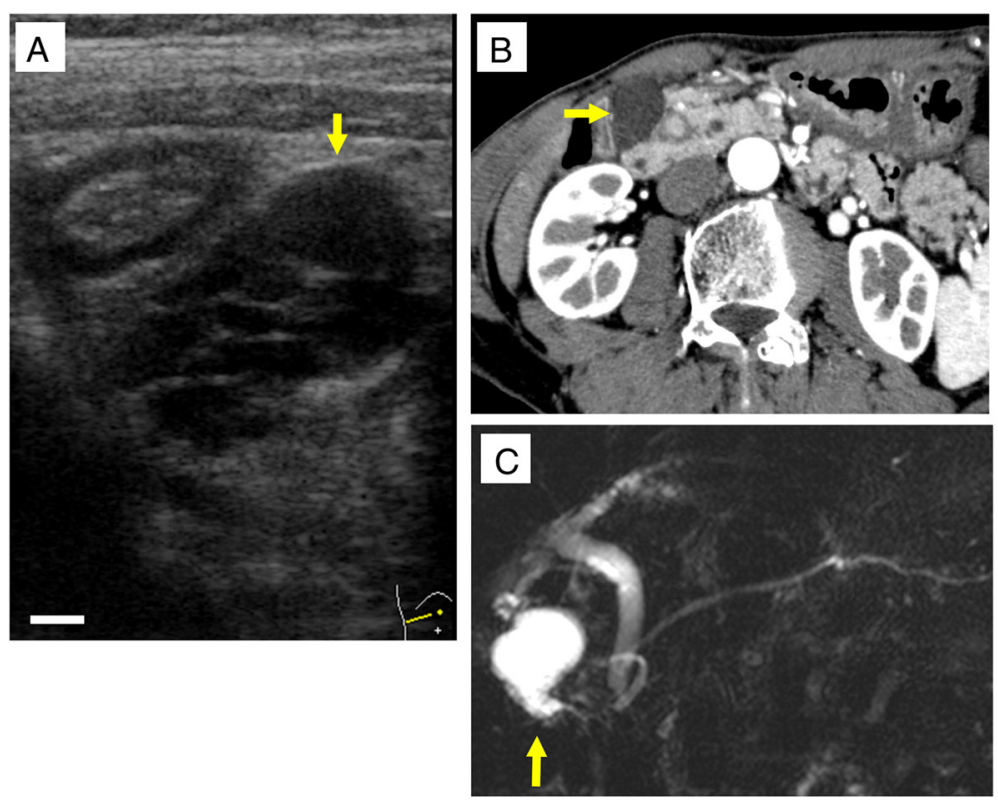

$\mathrm{D}$

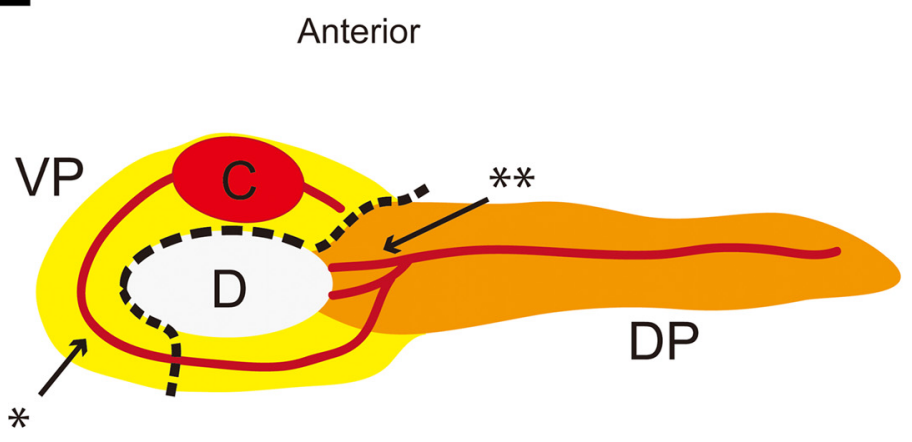

\section{Posterior}

Fig. 1 Diagnostic images of the intraductal papillary mucinous neoplasm (IPMN) in the patient's annular pancreas. a A multilocular cystic mass was detected by abdominal ultrasonography (arrow). The cystic mass was $30 \mathrm{~mm}$ in diameter. No intramural nodules or calcifications were present in the cystic lesion. Bar, $5 \mathrm{~mm}$. b Abdominal computed tomography revealed the presence of pancreatic tissue encircling the descending part of the duodenum (arrow). c Magnetic resonance cholangiopancreatography showed the annular duct encircling the duodenum. A 30-mm-diameter cystic mass was identified in the annular segment, and the mass was linked to the annular duct (arrow). The patient was preoperatively diagnosed with branch-duct-type IPMN (adenoma) with low-grade malignancy. $\mathbf{d}$ Schema of the annular pancreas in the present case. The yellow region indicates the ventral pancreas (VP). The orange region indicates the dorsal pancreas (DP). The red lines indicate the pancreatic ducts. The dotted line shows the resection line. Single asterisk shows the duct of Wirsung. Double asterisks show the duct of Santorini. C cystic mass, D duodenum

Universal 60-mm length and 3.5-mm staples, Covidien). The pancreas parenchym was gently compressed by the endostapler while compressed for $3 \mathrm{~min}$ while the preserving pancreatic capsule, and then it was transected slowly. The proximal pancreatic duct stump was closed with 4-0 nonabsorbable sutures in a transfixing pattern, and the pancreatic tissue stump was closed with 3-0 nonabsorbable sutures in a vertical mattress pattern (Fig. 2b, c). Finally, the dissected surface of the duodenum and the cut surface of the pancreas were covered by the upper jejunal wall (Fig. 2d, e). The operation time was $310 \mathrm{~min}$ and intraoperative blood loss was $60 \mathrm{~g}$. The patient's postoperative course was uneventful, and he was discharged on postoperative day 22 .

The resected specimen contained the cystic tumor with dilatation of the annular pancreatic duct (Fig. 3a). Histopathological analysis revealed that the cystic tumor was derived from the dilated pancreatic duct. The ductal epithelium had a papillary architecture comprising mucinrich columnar cells and high cellular density, but relatively low nuclear atypia (Fig. 3b, c). These findings were observed in both the main and branched pancreatic ducts. 

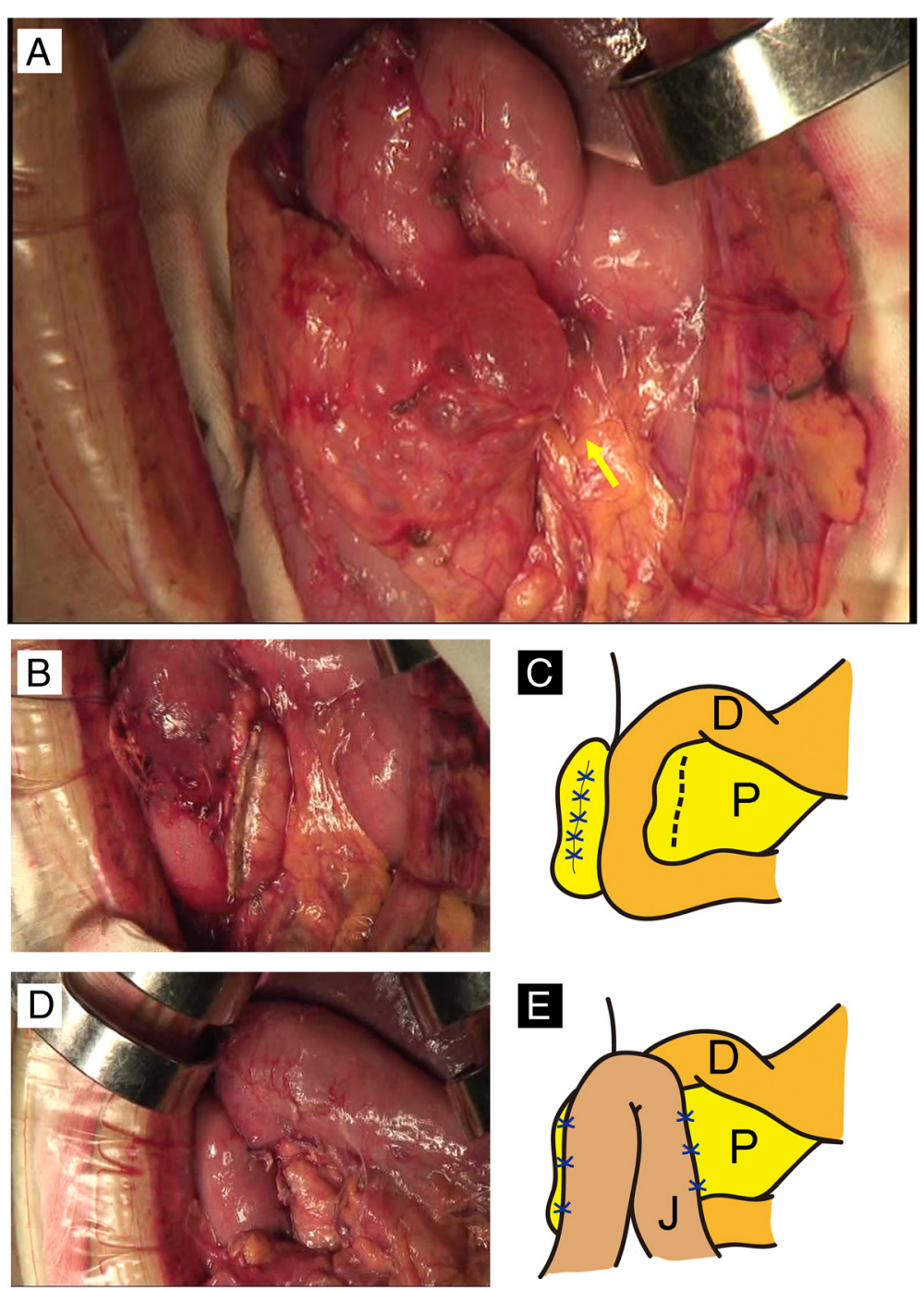

Fig. 2 Limited resection of the annular segment and jejunal patch. a The pancreatic tissue encircling the descending part of the duodenum was detected intraoperatively. The cystic mass was also found in the annular segment. $\mathbf{b}$, c The anterior annular segment was easily separated from the second portion of the duodenum. After resection of the annular segment, congestion of the separated surface of the duodenum was revealed. The schema of the appearance of the operative field after limited resection of the annular pancreas is demonstrated. $\mathbf{d}$, e The second portion of the duodenum and the pancreatic stump were covered by the jejunum to prevent duodenal leakage and pancreatic fistula formation. The schema of the appearance of the operative field after this procedure is demonstrated. $D$ duodenum, $P$ pancreas, $J$ jejunum

However, the surgical stump showed normal pancreatic tissue. Immunohistochemical analysis for p53 and Ki67 revealed less activity. Based on these results, the final diagnosis of the cystic tumor was an intraductal papillary mucinous adenoma with intermediated dysplasia in the annular pancreas. The patient had not developed recurrence 3 years after the resection.

\section{Discussion}

Annular pancreas is a rare congenital anomaly in which a ring of pancreatic tissue encircles the second portion of the duodenum. Lecco reported that in patients with imperfect rotation of the ventral pancreatic anlage, a ring of pancreatic tissue might be left around the second portion of the duodenum [3]. Baldwin reported that a persistent left ventral bud of the pancreas causes it to encircle the duodenum [4]. The most commonly accepted theory explaining the development of an annular pancreas is that proposed by Lecco [3]. The annular pancreas has a bimodal pattern of presentation; the first peak occurs in infancy, and a later peak occurs in the fourth decade of life [5]. Combined congenital anomalies in children include trisomy 21, cardiac anomalies, and intestinal malrotation. Pediatric patients usually present with gastrointestinal obstruction and jaundice. In contrast, adults do not commonly present with annular 

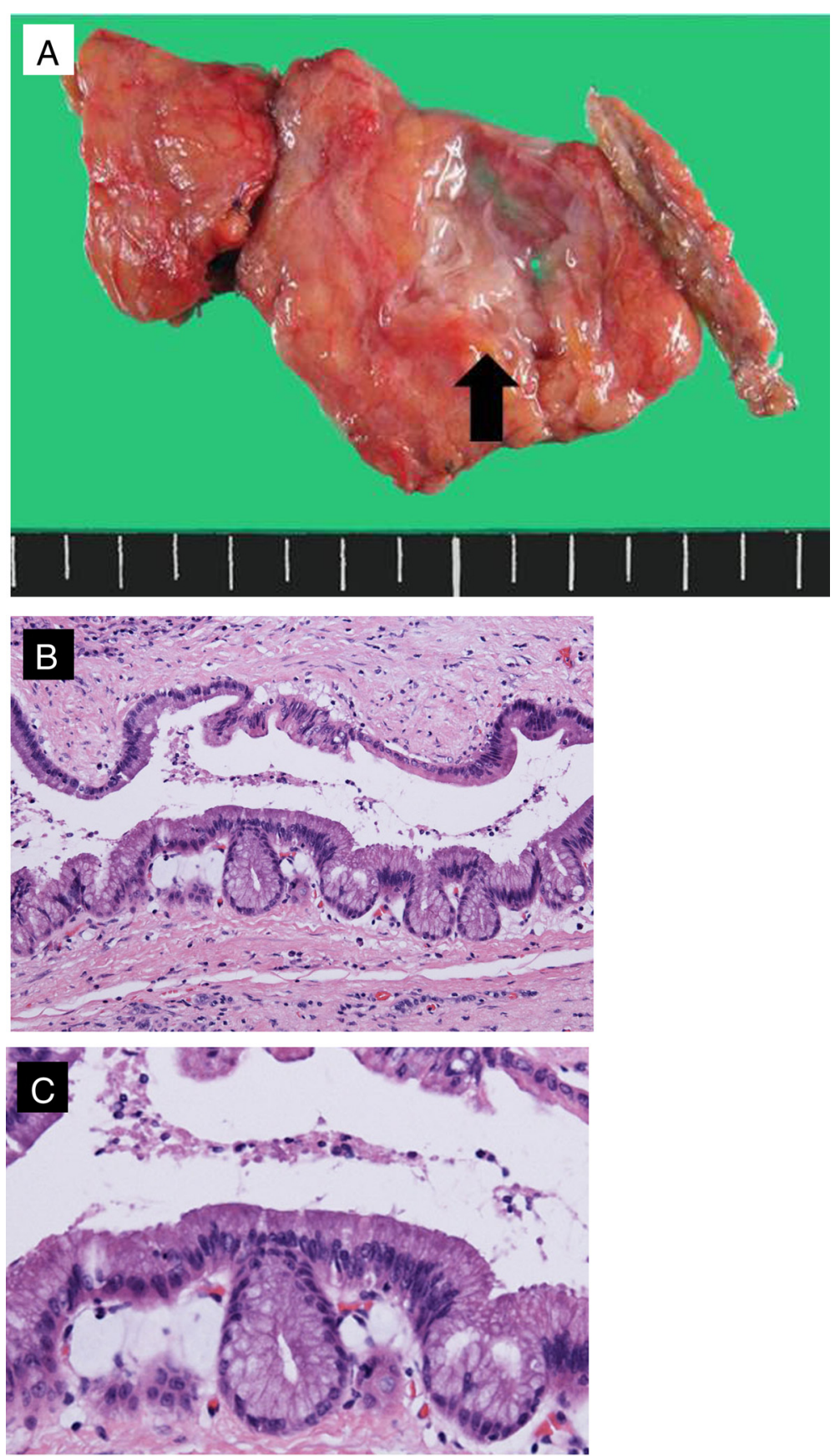

Fig. 3 Histopathological findings. a The resected specimen showed dilatation of the annular pancreatic duct. The scale resolution shows $5 \mathrm{~mm}$. Histopathological analysis showed a papillary architecture with nuclear atypia. b $\times 40$. $\mathbf{c} \times 200$

pancreas, although it is being identified with increasing frequency because of more liberal performance of abdominal computed tomography, magnetic resonance cholangiopancreatography, and endoscopic retrograde cholangiopancreatography [5]. In this case, the patient presented with epigastralgia. The reason of epigastralgia was caused by repeated mild pancreatitis. The patient complained of mild epigastralgia and showed mild increase of CA19-9 and serum amylase levels in his first visit to our hospital. Then, in the second visit, the patient had no symptoms and those two data showed normal range. With respect to combined neoplasms, Zyromski et al. reported that neoplasms developed in $24 \%$ of adults with an annular pancreas, and $48 \%$ of them were pancreatobiliary tumors [5]. Systemic search of PubMed for publications in English was 
performed. Search terms were "annular pancreas," "adult," and "neoplasm." Including our case, there are eight cases of pancreatic neoplasm associated with annular pancreas [6-12] (Table 1). The types of pancreatic neoplasm were as follows: four cases adenocarcinoma, two cases with mucinous cystic neoplasm, and one case with IPMN. The patients consisted of two males and six females with a mean age of $67.8 \pm$ 14.5 years. The neoplasms occurred in three cases of the annular segment and five cases of the dorsal segment. Six patients underwent surgical resections of pancreatic neoplasm associated with annular pancreas, including pancreaticoduodenectomy in two cases, total pancreatectomy in one case, distal pancreatectomy in one case, and limited pancreatic resection in one case. An anomalous pancreatic duct could cause the stasis of pancreatic juice and gives inflammatory injury to pancreatic ductal epithelium. An exposure of such chronic stimulation may induce oncogenic change to the ductal epithelium. Thus, pancreatobiliary tumors in patients with an annular pancreas attribute to chronic inflammatory changes associated with pancreatitis $[13,14]$. Thus, pancreatobiliary tumors in patients with an annular pancreas might be attributed to chronic inflammatory changes in this area associated with pancreatitis $[15,16]$. The present patient had continuous epigastric pain, and a cystic neoplasm was found in the annular segment of the pancreas.

IPMNs are characterized by cystic dilation of the main and/or branched pancreatic ducts and intraductal proliferation of neoplastic mucinous cells arranged into papillary structures $[17,18]$. These tumors have a wide spectrum of atypical grades ranging from low-grade dysplasia to invasive carcinoma [19]. The diagnosis of IPMN of the pancreas has markedly increased in the last few decades because of the widespread use of high-resolution imaging [20, 21]. IPMNs are classified as main duct type, branch duct type, and combined type, according to the area of involvement of the pancreatic ductal system [22, 23]. The present patient had a branch duct type of IPMNs (adenoma) with low-grade malignancy [22, 23]. However, the patient experienced abdominal pain, and the diameter of the tumor was large $(30 \mathrm{~mm})$. Although there is a controversy regarding whether pancreatic resection or close follow-up should be performed to treat IPMNs with lowgrade malignant potential, especially in cases similar to the present case, we performed surgical extirpation of the tumor according to the Sendai consensus guidelines [21-26].

Various operative procedures are available to treat IPMNs with a low risk of malignancy [27-30]. Nakagohri et al. reported good surgical outcomes for noninvasive or minimally invasive IPMNs after inferior pancreatic head resection [31]. In this procedure, the uncinate process and pancreatic parenchyma around the duct of Wirsung are resected, preserving the pancreatic head around the duct of Santorini [31]. Takada described ventral pancreatectomy, which involves resection of only the ventral segment of the pancreas, preserving the dorsal segment and the main pancreatic duct [32]. Of course, division of the annular segment is generally not recommended because of the high incidence of postoperative complications such as fistula formation, pancreatitis, pancreatic laceration, and/or recurrent duodenal stenosis secondary to local fibrosis [5, 33, 34]. Thus, in the present case, the resection area was very carefully determined, and an additional procedure was performed to avoid postoperative complications.

In many cases, the pancreatic annulus cannot be separated because of the dense adhesion between the duodenum and annulus [2]. In our case, however, the adhesion between the annular segment and second portion of duodenum was fortunately loose, and we easily ligated the annular pancreatic duct. The annular segment (including the tumor) was removed, and the pancreatic head around the duct of Santorini was preserved. Additionally, the second portion of the duodenum and the pancreatic stump were covered by the jejunum to prevent duodenal leakage and pancreatic fistula formation. The present case suggests that limited annular pancreatic resection is safe when the adhesion between the duodenum and annular segment is loose and the locations of the annular pancreatic duct and the duct of Santorini are definitively identified. However, the precise indications for this procedure remain to be elucidated.

Table 1 The literature of pancreatic neoplasm associated with annular pancreas

\begin{tabular}{lllllll}
\hline Author & Year & Age & Gender & Origin & Pathology & Surgical treatment \\
\hline Matsusue et al. [6] & 1984 & 53 & F & AP & Adenocarcinoma & Total pancreatectomy \\
Kamisawa et al. [8] & 1995 & 79 & F & DP & Adenocarcinoma & None \\
Yasui et al. [7] & 1995 & 54 & M & AP & Adenocarcinoma & Pylorus-preserving pancreaticoduodenectomy \\
Ben-David et al. [9] & 2004 & 52 & F & DP & Adenocarcinoma & Pancreaticoduodenectomy \\
Cholet et al. [10] & 2004 & 88 & F & DP & Adenocarcinoma & None \\
ljichi et al. [11] & 2009 & 60 & F & DP & MCN & Pancreaticoduodenectomy \\
Milone et al. [12] & 2013 & 78 & F & DP & MCN & Distal pancreatectomy \\
Our case & 2015 & 78 & M & AP & IPMN & Limited pancreatic resection \\
\hline
\end{tabular}

$M C N$ mucinous cystic neoplasm, IPMN intraductal papillary mucinous neoplasm, $A P$ annular pancreas, $D P$ dorsal pancreas 


\section{Conclusions}

In conclusion, the possibility of coexisting pancreatobiliary disorders such as IPMNs should be kept in mind in adult patients with an annular pancreas. Partial resection of the pancreas, including division of the annular segment, may be safe and effective in selected patients.

\section{Consent}

Written informed consent was obtained from the patient for publication of this case report and any accompanying images.

\section{Competing interests}

The authors declare that they have no competing interests.

\section{Authors' contributions}

SK is the first author and was the main surgeon in this case. He played the main role in writing and editing the paper. YK was the chief clinician in this case and mainly supported SK in performing the operation. He also edited the entire content of the main body of the paper. YN suggested the feasibility of procedure before the operation and edited the entire content of the main body of the paper. MI pathologically diagnosed the IPMN and elucidated the pathological features in this case. HF supervised the study and approved the final submission of this article. All authors read and approved the final manuscript.

\section{Acknowledgements}

The authors thank Amane Kitasato, Tomohiko Adachi, and Shinya Onizuka for their constructive advice.

\section{Author details}

${ }^{1}$ Department of Surgery, National Hospital Organization Nagasaki Medical Center, Ohmura, Nagasaki, Japan. ²Department of Pathology, National Hospital Organization Nagasaki Medical Center, Ohmura, Nagasaki, Japan.

\section{Received: 4 February 2015 Accepted: 7 August 2015}

\section{Published online: 25 August 2015}

\section{References}

1. Tiedemann F. Uber die Verschiedenheiten des Ausfuhrungsganges der Bauchspeicheldruse bei den Menschen und Saugetieren. Dtsch Arch Physiol. 1818;4:403

2. Ravitch MM, Woods Jr AC. Annular pancreas. Ann Surg. 1950;132(6):1116-27.

3. Lecco T. Zur morphologie des pancreas annulare. Sitzungberichte de Kaiserlichen Akademie der Wissenschaften. 1910;69:391-406.

4. Baldwin WA. A specimen of annular pancreas. Anat Rec. 1910;4(8):299-304.

5. Zyromski NJ, Sandoval JA, Pitt HA, Ladd AP, Fogel EL, Mattar WE, et al. Annular pancreas: dramatic differences between children and adults. J Am Coll Surg. 2008;206(5):1019-25. discussion 25-7.

6. Matsusue S, Kashihara S, Koizumi S. Pancreatectomy for carcinoma of the head of the pancreas associated with multiple anomalies including the preduodenal portal vein. Jpn J Surg. 1984;14(5):394-8.

7. Yasui A, Nimura Y, Kondou S, Kamiya J. Duodenal obstruction due to annular pancreas associated with pancreatic head carcinoma. Hepatogastroenterology. 1995;42(6):1017-22

8. Kamisawa T, Tabata I, Isawa T, Ishiwata J, Fukayama M, Koike M. Annular pancreas associated with carcinoma in the dorsal part of pancreas divisum. Int J Pancreatol. 1995:17(2):207-11.

9. Ben-David K, Falcone Jr RA, Matthews JB. Diffuse pancreatic adenocarcinoma identified in an adult with annular pancreas. J Gastrointest Surg. 2004;8(5):565-8.

10. Cholet F, Bideau K, Nonent M, Nousbaum JB, Gouerou H, Robaszkiewicz M. Coexistence of annular pancreas with carcinoma in the dorsal part of pancreas divisum: diagnostic value of magnetic resonance cholangiopancreatography. Abdom Imaging. 2004;29(6):703-6.

11. ljichi $H$, Nishizaki $T$, Terashi $T$, Shiraishi $T$, Takahashi I, Wada $H$, et al. Coexistence of mucinous cystic neoplasm occurring in the head of the pancreas with annular pancreas: report of a case. Surg Today. 2009;39(10):897-900
12. Milone L, Okhunov Z, Gumbs AA. Laparoscopic diagnosis of annular pancreas in a patient with mucinous cystoadenoma of the body of the pancreas. J Gastrointest Cancer. 2012;43(2):367-9.

13. Traverso LW, Kozarek RA, Simpson T, Galagan KA. Pancreatic duct obstruction as a potential etiology of pancreatic adenocarcinoma: a clue from pancreas divisum. Am J Gastroenterol. 1993;88(1):117-9.

14. Nishino T, Toki F, Oi I, Oyama H, Hatori T, Shiratori K. Prevalence of pancreatic and biliary tract tumors in pancreas divisum. J Gastroenterol. 2006:41(11):1088-93.

15. Adachi T, Tajima Y, Kuroki T, Mishima T, Kitasato A, Fukuda K, et al. Bile-reflux into the pancreatic ducts is associated with the development of intraductal papillary carcinoma in hamsters. J Surg Res. 2006;136(1):106-11.

16. Lahmar A, Abid SB, Arfa MN, Bayar R, Khalfallah MT, Mzabi-Regaya S. Metachronous cancer of gallbladder and pancreas with pancreatobiliary maljunction. World J Gastrointest Surg. 2010;2(4):143-6.

17. Sadakari Y, lenaga J, Kobayashi K, Miyasaka Y, Takahata S, Nakamura M, et al. Cyst size indicates malignant transformation in branch duct intraducta papillary mucinous neoplasm of the pancreas without mural nodules. Pancreas. 2010;39(2):232-6.

18. Ohno E, Itoh A, Kawashima $H$, Ishikawa $T$, Matsubara $H$, Itoh $Y$, et al. Malignant transformation of branch duct-type intraductal papillary mucinous neoplasms of the pancreas based on contrast-enhanced endoscopic ultrasonography morphological changes: focus on malignant transformation of intraductal papillary mucinous neoplasm itself. Pancreas. 2012;41(6):855-62.

19. Thompson R. Cancer: epithelial subtype influences the prognosis of invasive IPMN. Nat Rev Gastroenterol Hepatol. 2011;8(8):420.

20. Gourgiotis S, Ridolfini MP, Germanos S. Intraductal papillary mucinous neoplasms of the pancreas. Eur J Surg Oncol. 2007:33(6):678-84.

21. Anand N, Sampath K, Wu BU. Cyst features and risk of malignancy in intraductal papillary mucinous neoplasms of the pancreas: a meta-analysis. Clin Gastroenterol Hepatol. 2013:11(8):913-21. quiz e59-60.

22. Tanaka M, Chari S, Adsay V, Fernandez-del Castillo C, Falconi M, Shimizu M, et al. International consensus quidelines for management of intraductal papillary mucinous neoplasms and mucinous cystic neoplasms of the pancreas. Pancreatology. 2006;6(1-2):17-32.

23. Tanaka M, Fernandez-del Castillo C, Adsay V, Chari S, Falconi M, Jang JY, et al. International consensus guidelines 2012 for the management of IPMN and MCN of the pancreas. Pancreatology. 2012;12(3):183-97.

24. Fritz S, Klauss M, Bergmann F, Strobel O, Schneider L, Werner J, et al. Pancreatic main-duct involvement in branch-duct IPMNs: an underestimated risk. Ann Surg. 2014;260(5):848-56.

25. Tanaka M. Controversies in the management of pancreatic IPMN. Nat Rev Gastroenterol Hepatol. 2011;8(1):56-60.

26. Talukdar R, Nageshwar RD. Treatment of pancreatic cystic neoplasm: surgery or conservative? Clin Gastroenterol Hepatol. 2014;12(1):145-51.

27. Miller JR, Meyer JE, Waters JA, Al-Haddad M, Dewitt J, Sherman S, et al. Outcome of the pancreatic remnant following segmental pancreatectomy for non-invasive intraductal papillary mucinous neoplasm. HPB (Oxford). 2011;13(11):759-66.

28. Fujii T, Kanda M, Kodera Y, Nagai S, Sahin T, Kanzaki A, et al. Comparison of pancreatic head resection with segmental duodenectomy and pyloruspreserving pancreatoduodenectomy for benign and low-grade malignant neoplasms of the pancreatic head. Pancreas. 2011:40(8):1258-63.

29. Nakao A, Fernandez-Cruz L. Pancreatic head resection with segmental duodenectomy: safety and long-term results. Ann Surg. 2007;246(6):923-8. discussion 9-31.

30. Place TL, Nau P, Mezhir JJ. Minimally invasive pancreatectomy for cancer: a critical review of the current literature. J Gastrointest Surg. 2014;19(2):375-86.

31. Nakagohri T, Kinoshita T, Konishi M, Takahashi S, Gotohda N, Kobayashi S, et al. Inferior head resection of the pancreas for intraductal papillary mucinous neoplasms. J Hepatobiliary Pancreat Sci. 2010;17(6):798-802.

32. Takada T. Ventral pancreatectomy: resection of the ventral segment of the pancreas. J Hepatobiliary Pancreat Surg. 1993;1(1):36-40.

33. Ohno Y, Kanematsu T. Annular pancreas causing localized recurrent pancreatitis in a child: report of a case. Surg Today. 2008;38(11):1052-5.

34. Ladd AP, Madura JA. Congenital duodenal anomalies in the adult. Arch Surg. 2001;136(5):576-84. 